\title{
Risk Behaviors of Dominican Adolescents in Their Homeland and in the United States
}

Lynn M. Babington

Fairfield University, Ibabington@fairfield.edu

B. R. Kelley

C. A. Patsdaughter

Follow this and additional works at: https://digitalcommons.fairfield.edu/nursing-facultypubs

Copyright 2007 Elsevier

NOTICE: This is the author's version of a work that was accepted for publication in Journal of Pediatric Health Care. Changes resulting from the publishing process, such as peer review, editing, corrections, structural formatting, and other quality control mechanisms may not be reflected in this document. Changes may have been made to this work since it was submitted for publication. A definitive version was subsequently published in Journal of Pediatric Health Care, 21(6), 2007.DOI: org/10.1016/j.pedhc.2007.03.005

\section{Peer Reviewed}

\section{Repository Citation}

Babington, Lynn M.; Kelley, B. R.; and Patsdaughter, C. A., "Risk Behaviors of Dominican Adolescents in Their Homeland and in the United States" (2007). Nursing and Health Studies Faculty Publications. 16. https://digitalcommons.fairfield.edu/nursing-facultypubs/16

\section{Published Citation}

Babington, L.M., B.R. Kelley, \& Patsdaughter, C.A. (2007). Risk Behaviors of Dominican Adolescents in Their Homeland and in the United States. Journal of Pediatric Health Care, 21(6), 372-380.

This item has been accepted for inclusion in DigitalCommons@Fairfield by an authorized administrator of DigitalCommons@Fairfield. It is brought to you by DigitalCommons@Fairfield with permission from the rightsholder(s) and is protected by copyright and/or related rights. You are free to use this item in any way that is permitted by the copyright and related rights legislation that applies to your use. For other uses, you need to obtain permission from the rights-holder(s) directly, unless additional rights are indicated by a Creative Commons license in the record and/or on the work itself. For more information, please contact digitalcommons@fairfield.edu. 


\title{
Risk Behaviors of Dominican Adolescents \\ in Their Homeland and in the United States
}

\begin{abstract}
Introduction: Adolescence is a time of developmental change: biological, cognitive, and psychosocial. The major task of adolescence is to become an independent individual capable of functioning as a contributing adult within culturally expected norms. As exciting and challenging as this time can be for adolescents, it is often accompanied by frustration and difficulties with adjustments. One typical response of adolescents to these changes is engaging in high risk behaviors. The purpose of this study was to compare self-reported high risk behaviors that may lead to unintentional and intentional injuries; suicide-related risks; and risks associated with smoking, alcohol, and drug use among adolescents living in the Dominican Republic (D.R) and Dominican adolescents living in the United States (U.S.).

Methods: This study used a cross-sectional, comparative design with data collection sites in Las Matas de Farfan, D.R. and Boston, U.S. Data were collected using the 99-item 1999 Youth Risk Behavior Survey (YRBS).

Results: Findings indicted that adolescents in both groups reported similar risk behaviors in all areas; however, there were few differences between the two groups that did exist.

Discussion: Findings support the view that risk taking is a universal practice among youth. To educate youth regarding outcomes of high risk behaviors, it is necessary for providers to engage in discussion with adolescents regarding specific risk behaviors that are commonly practiced in their homeland or in their cultural groups. The need for culturally competent care is especially important with immigrant youth who are not only struggling with identity formation but doing so in a new culture.
\end{abstract}


Adolescence, the common term used to describe the transition from childhood to adulthood, occurs in every culture. It is a time of developmental change: biological, cognitive, and psychosocial. The major task of adolescence is to become an independent individual capable of functioning as a contributing adult within culturally expected norms. As exciting and challenging as this time can be for adolescents, it is often accompanied by frustration and difficulties with adjustments. One typical response of adolescents to these changes is engaging in high risk behaviors. Research has shown that adolescent risk behaviors are functional, purposive, instrumental, and goal-directed (Jessor, 1992; Le Breton, 2004). While specific high risk behaviors may be cultural bound, the impetus for the adolescent to behave this way is a universal trait across cultures (Bronfenbrenner, 1979; Lightfoot, 1997; Rice, Dolgin, \& Dolgin, 2001). The purpose of this study was to compare self-reported high risk behaviors that may lead to untentional and intentional injuries; suicide-related risk behaviors; and smoking, alcohol, and marijuana risk behaviors among adolescents living in the Dominican Republic (D.R) and first and second generation Dominican adolescents living in the United States (U.S.). Comparative findings on sexual risk behaviors have been previously reported (Patsdaughter, Kelley, Babington, \& Dyer, 2005).

During the 10-year period from 1993 through 2002, the greatest number of immigrants to Massachusetts came from China, the former Soviet Union, the Dominican Republic, Haiti, India, and Vietnam. Together, immigrants from these countries accounted for more than two-fifths (42.5\%) of total admissions (Federation for American Immigration Reform (FAIR), 2004). In 2003, 26,205 Dominicans immigrated to the U.S. and comprised 3.7\% of all immigrants entering this country (U.S. Census Bureau, 2004). 
Hispanics and Hispanic subpopulations trail non-Hispanic Whites on various measures of health including lack of health insurance, regular source of ongoing health care, and prenatal care in the first trimester; they also have an increased number of deaths resulting from homicide. Poor health outcomes for Hispanics include higher infant mortality rates, greater prenatal exposure to human immunodeficiency virus (HIV), an increase in teen birth rates, and an increase in tobacco use compared to Whites (Centers for Disease Control (CDC), 2004b). Many of these disparities can be attributed to unfamiliarity with the U.S. health care system, lack of access to services, and cultural attitudes about the use of traditional versus western health care. However, health disparities also result from high risk behaviors, which comprise a unique aspect of adolescent growth and development across cultures.

Risk-taking behavior, a hallmark of adolescence, is an essential activity in the transition to adulthood. There are three dominant theoretical views of adolescent risk-taking behaviors: biological, psychological, and universal. The biologically-based theorists attribute adolescent risk taking behaviors to genetic predispositions as well as hormonal and psychosocial changes that occur during puberty (Blum, McNeely, \& Nonnemaker, 2001; Ingra \& Irwin, 1996). Theorists who subscribe to a psychological view postulate that sensation seeking, based on a need for varied, novel, and complex experiences, drives adolescents to take physical and social risks (Weinstein, 1984; Zukerman, 1979). Universal theorists consider biologic and genetic factors as well as cultural and environmental factors as having an influence on adolescent behavior. This view assumes the existence of universal features of human development and mental health across different ethnic and cultural groups. The belief is that observable expressions of emotion and the manifestations of mental illness are shaped by the environment and/or culture. The universal orientation to understanding human behavior emphasizes the interaction between universal features and cultural 
and/or environmental influences (Choi, 2002). The study reported in this paper was based on a universal view of adolescent behavior.

Culture and ethnicity affect both personality and surrounding social conditions. These dual factors are important determinants in the prevalence of adolescent risk-taking behavior which has been reported across cultures (Gabhainn \& Francois, 2000, Hibell et al., 2000). A study that compared HIV risk behaviors of U.S. Hispanic and Dominican Republic youth demonstrated that adolescents in both countries were at risk but with different mediating factors (Westhoff, McDermott, \& Holcomb, 1996) . Blake, Ledsky, Goodenow, and O’Donnell (2001) found that immigrant youth living in the U.S. for 6 years or less reported lower lifetime and recent alcohol and marijuana use than immigrant youth who lived here longer. They also found that recent immigrants were most likely to report peer pressure to engage in risk behavior and less parental support to avoid risky behaviors. Acculturation also plays a part in influencing adolescent high risk behaviors. The more acculturated a new immigrant is, the greater his or her risk for engaging in high risk behaviors. A study that examined acculturation in adolescents and adults aged 15 to 54 found that acculturation items predicted greater risk of having any DSM-III-R disorders for Mexican-Americans and "other" Hispanic groups and greater risk of having a substance abuse disorder for Puerto Ricans (Ortega, Rosenheck, Alegria, \& Desai, 2000).

Both the European School Survey Project on Alcohol and Other Drugs (ESPAD) (Hibell et al., 2003) and the Health Behavior in School-Age Children (HBSC) survey (Currie, 1998) indicated the importance of cultural background when studying high-risk behaviors in adolescents. Findings from these studies supported the view that while culture and ethnicity may influence the types of high risk behaviors in which adolescents engage, risk-taking appears to be a universal experience. Vazsonyi, Trejos-Castillo, and Huang (2006) believed that Self-Control Theory was a key factor in 
understanding adolescent health-compromising behaviors. Self-control develops during childhood mostly from socialization influences and pressures both at home and school. If these pressures are missing, low self control results, which paves the way for engaging in high risk behavior. Research results were consistent with the Self Control Theory: low self-control was associated with measures of health-compromising behaviors in a variety of national, state, and local samples (Eaton et al., 2006). Bradley and Wildman (2002) focused on two psychosocial predictors of adolescent risk and reckless behaviors: sensation seeking and peer pressure. These researchers found that risk behaviors (i.e., socially approved adventurous and thrill-seeking acts such as motorcycle riding, mountaineering, and bungee jumping) were reliably predicted by sensation seeking but not by peer pressure, whereas peer pressure was a significant predictor of reckless behaviors such a substance abuse, reckless driving, and sexual behaviors.

The researchers' interest in Dominican adolescents evolved from working with Dominican families in Boston who have recently immigrated from the D.R. and working with adolescents in the D.R. . For the past 10 years, two of the researchers have been involved in a volunteer nursing organization, Intercultural Nursing, Inc. (INI). INI sponsors trips twice a year to the D.R. to provide primary health services in rural campos (i.e., villages) on the western frontier of the country. Understanding the dynamics of adolescent behaviors offers health care providers a window of opportunity for both intervention and education regarding the consequences of such behaviors. This is especially true with adolescents from another culture who move to the U.S. In light of the U.S. Public Health Service Year 2010 goals that specifically target adolescents for special risk reduction efforts(CDC, 2004a), this information will be useful to both health care providers and educators to help new and recent immigrant adolescents understand the dangers of these selected risk behaviors, particularly as they acculturate to living in the U.S. 
Methods

\section{Design and Setting}

This study used a cross-sectional, comparative design with data collection sites in Las Matas de Farfan, D.R. and Boston, U.S. Data collection in the D.R. took place primarily in government sponsored schools. Data were collected by the investigators during annual 2-week trips in which

heath care clinics were set up in rural campos by groups of volunteer nurses (Intercultural Nursing, Inc.) to provide primary health care. Most of the study participants were recruited from a local government sponsored high school. The investigators approached the high school principal and described the purpose of the study. The principal informed students of the opportunity to participate in the study, and students stayed after school to complete the questionnaire. As a small incentive for participation, students received a bandana that sported the Dominican flag. Data collection in the U.S. took place in the Boston area primarily at youth group activities at the Boys and Girls Club and YMCA and events in urban community settings such as street fairs and youth sporting events. A face-to-face recruitment strategy was used. The investigators described the purpose of the study and offered a small incentive for participation (bandana with Dominican flag). Snowball sampling occurred as peers also recruited their friends to participate

The Northeastern University Institutional Review Board for the Protection of Human Subjects (IRB) approved the study. A waiver of parental consent was obtained from the IRB at Northeastern University after the investigators presented the following explanations on why parental consent should not be required: 1) The YRBS is routinely administered in high schools in the US without explicit parental consent, 2) Parents in the DR would likely refuse to sign and be intimidated by an official document since parental consent is not a familiar practice in the DR; and furthermore, many adults in the DR are illiterate, 3 )It would be unlikely that adolescents in either 
the DR or the US would be willing to honestly answer questions on risk behaviors, particularly sexual behaviors if they needed to obtain their parents consent. Although parental consent was not required, adolescent assent was obtained by their willingness to complete the survey. Students were told that their responses were anonymous, their participation was voluntary, and no names or other personal identifiers were requested.

Instruments

Data were collected using three survey instruments: the 99-item 1999 Youth Risk Behavior Survey (YRBS) (Brener 2004), Rosenberg Self Esteem Scale (RSES) (Rosenberg, 1965), and the Adolescent Social Influence Scale (ASIS), which was specifically developed for this study. This paper presents risk findings from the YRBS.

\section{Youth Risk Behavior Survey}

The YRBS has been widely used and methodologically tested with numerous, culturally diverse populations (Brener et al., 2004; Brener et al., 2002; Kann et al., 2000). The CDC developed the Youth Risk Behavior Surveillance System (YRBSS) in order to identify priority health-risk behaviors among youth, assess whether the prevalence of these self-reported behaviors change over time, and provide data for comparison among different geographic samples. Review of the leading causes of morbidity and mortality among young people led to the categorization of health-risk behaviors into six areas: (a) behaviors resulting in unintentional and intentional injuries; (b) tobacco use; (c) alcohol and other drug use; (d) sexual behaviors that contribute to unintended pregnancy and disease; (e) unhealthy dietary behaviors; and (f) physical inactivity. The YRBSS is included in national, state, and local school-based surveys and national household-based surveys of high school students. The national school-based surveys are conducted biannually in odd-numbered years (CDC, 2006). 
YRBS results are based on self-reported data. Validation of self-reported high-risk behaviors is limited; however, data collected from school-based surveys have been consistent with data from other youth surveys. Brener, Collins, Kann, Warren, and Williams (1995) evaluated the reliability of self-reported health risk behaviors measured by the YRBS by conducting a test-retest reliability study with 1679 students and found that item reliability estimates for $72 \%$ of the 53 items were "substantial" (i.e., 61-80\%) or higher.

The CDC developed two versions of the YRBS for Spanish-speaking students: the Southwestern U.S. version and a Southeastern-Puerto Rican version. Westhoff et al. (1996) tested the Southeastern-Puerto Rican version in the D.R. for appropriateness, language, and cultural sensitivity. They noted that the true cultural specificity of this Spanish version for Dominican youth is unknown and identified this lack of information as a potential limitation of their study. The Southeastern-Puerto-Rican version was administered to the Dominican youth in this study. However, it should be noted that the Southeastern-Puerto Rican version may contain cultural constructs and colloquial language that differ from the Spanish used in the D.R. All items of the YRBS were reviewed for appropriateness and clarity for this study by two bilingual Dominican nurse practitioner students. Items and responses were presented on the questionnaire in both English and Caribbean Spanish.

\section{Rosenberg Self Esteem Survey}

The Rosenberg Self Esteem Scale (RSES) first published in 1965 was one of the earliest measures of overall self-esteem. According to Rosenberg, self-esteem is a variable reflecting the general attitude a person has about personal value and this global attitude is what the scale intended to assess. The 10-item Rosenberg Self Esteem Scale used measures global self esteem on a 4-point Guttman-like scale. Possible scores range from 0-10, with 10 indicating very high self-esteem (Rosenberg, 1965, 
1989). It has demonstrated reliability in both English and Spanish (with Mexican and Dominican adolescents) with reported internal consistency ranging from .64 to .88 (Banos \& Guillen, 2000; Orshan, 1999; Joiner \& Kashubeck, 1996; Fleming \& Courner, 1980).

\section{Statistical Analysis}

Prior to data analysis, each questionnaire was visually inspected for patterned responses such as choosing the same answer for each question and extreme response set bias as well as excessive missing data. None of the questionnaires were excluded based on these criteria. Data analyses were conducted using the Statistical Package for the Social Sciences (SPSS) (SPSS, 2005) and Chi-Square tests were the statistic used to test for differences in risk behaviors between the D.R. and U.S. subsamples. Risk behaviors that could lead to unintentional and intentional injuries were dichotomized into low risk (e.g., always wear a motorcycle helmet, never carry a gun) and high risk (e.g., carried a weapon 1 through six or more days in the past 30 days).

\section{Sample Demographic Characteristics}

Survey data were collected from a convenience sample of 390 Dominican adolescents, including 192 adolescents in the D.R. and 198 adolescents in the U.S. (see Table 1). Participants ranged in age from 12 to 18 years. The two subsamples differed significantly by age, with proportionately fewer adolescents in the D.R. in younger age groups than adolescents in the U.S. More participants in the D.R. subsample were older (i.e., $52.1 \%$ were age 17 or older) than participants in the U.S. subsample (i.e., $33.9 \%$ were age 17 or older). Likewise, there were proportionately fewer adolescents in the D.R. in the 9th or 10th grade, whereas proportionately more adolescents in the U.S. reported that they were not currently in school. There were more males than females in the U.S. subsample, but the two groups did not significantly differ by gender. 
Dominican adolescents in the U.S. were a fairly acculturated group as indicated by the fact that twothirds $(n=132,66.6 \%)$ had lived in this country for more than 6 years.

\section{Findings}

Findings from the YRBS will be reported in four major categories: risk behaviors that could lead to unintentional injury; risk behaviors that could lead to intentional injuries; suicide-related risk behaviors; and smoking, alcohol, and marijuana use. Findings are reported in Tables 2 through 5.

\section{Unintentional Injury Risk Behaviors}

There were no significant difference between the D.R. and U.S. subsamples in motorcycle helmet use, seatbelt use, and riding in a car that was driven by someone who was drinking. However, over two fifths ( $\mathrm{n}=80,41.7 \%)$ of the D.R. subsample were at high risk for an unintentional injury resulting from riding a motorcycle without a helmet, whereas over three-fourths of the U.S. subsample reported that motorcycle helmet use was not applicable, which likely meant that they did not ride motorcycles at all. These findings were consistent with the investigators' observations that motorcycles are prevalent means of transportation in the D.R., and few Dominicans consistently wear helmets since there is no law requiring helmet use. A majority of adolescents in both the D.R. and U.S. were at low risk for unintentional injuries from not wearing seatbelts while riding in a car or from riding in a vehicle that was driven by someone who had been drinking.

Proportionately more adolescents in the D.R. $(n=28,14.6 \%)$ were at risk for unintentional injuries due to driving a car or other vehicle when they had been drinking than adolescents in the U.S. $(n=17,8.6 \%)$, and this difference was significant $\left(\chi^{2}(1, N=383)=3.80, p<.05\right)$. Underage drinking is less regulated in the D.R. than in the U.S., and adolescents in the D.R. frequent discos/bars and pizza parlors where alcohol is served.

\section{Intentional Injury Risk Behaviors}


Proportionately more Dominican youth in the U.S. $(n=30,15.2 \%)$ reported that they carried a weapon such as a gun, knife, or club than their counterparts in the D.R. $(n=11,5.7 \%)\left(\chi^{2}(1\right.$, $N=383)=3.68, p<.005)$, which placed U.S. adolescents at higher risk for intentional injuries. This difference is likely due to the availability of weapons in the U.S.

No significant differences were found between the D.R. and U.S. subsamples in their risk for intentional injuries related to being involved in a physical fight in the past 12 months or being pushed, slapped, punched, or hurt on a date. Only about a third of the adolescents in both subsamples reported involvement in physical fights, and even smaller percentages (6.3\% in the D.R. and $6.6 \%$ in the U.S.) reported date violence including unwanted sexual activity.

\section{Suicide-Related Risks}

The only significant difference between the two subsamples with respect to suicide-related risks was in response to the question, "During the past 12 months, did you ever feel so sad or hopeless almost every day for two weeks or more in a row that you stopped doing some usual activities?". Proportionately more adolescents in the D.R. subsample $(n=43,22.4 \%)$ reported they had these experiences than their counterparts in the U.S. subsample $(n=32,16.2 \%)\left(\chi^{2}(1, N=385)\right.$ $=2.86, p<.05)$. There were no significant differences between the two subsamples on whether or not participants seriously considered attempting suicide, made a suicide plan, or attempted suicide in the past 12 months.

Smoking, Alcohol, and Marijuana Risk Behaviors

A significant difference was found between the two subsamples on the item that addressed whether or not the respondents ever tried smoking; proportionately more Dominican adolescents in the U.S. subsample $(n=71,35.9 \%)$ than adolescents in the D.R. $(n=48,25 \%)$ reported that they ever tried smoking cigarettes $\left(\chi^{2}(1, N=384)=4.53, p<.05\right)$. However, there were no significant 
differences between the two subsamples with respect to smoking regularly (i.e., smoking at least one cigarette a day for 30 days).

There was no significant difference between the two subsamples in the proportion of adolescents who reported that they ever drank alcohol. However, proportionately more adolescents in the D.R. subsample $(n=59,30.7 \%)$ reported that they had five or more drinks in a row within the past 30 days than adolescents in the U.S. subsample $(n=40,20.2 \%)\left(\chi^{2}(1, N=380)=6.38, p<\right.$ $.01)$. In contrast, over a fifth $(n=43,21.7 \%)$ of adolescents in the U.S. subsample reported that they ever used marijuana, while only a few $(n=4,2.1 \%)$ adolescents in the D.R. reported marijuana use $\left(\chi^{2}(1, N=380)=35.53, p<.0001\right)$.

It is noteworthy for all risk behavior items with the exception of suicide attempt in the past 12 months, there were more missing or "not reported" data among adolescents in the D.R. subsample than among adolescents in the U.S. subsample. A probable explanation for this trend is that students in the D.R. are not as exposed to surveys eliciting their behaviors and they may have left responses blank (vs. choosing the "not applicable" option) because they had never experienced the behavior in question. However, in contrast to availability of objects or substances such as guns or marijuana, suicide is a potential risk for all adolescents regardless of circumstances.

\section{Discussion}

This study was designed to assess any differences between Dominican adolescents and first and second generation Dominican adolescents in the U.S. in risk behaviors for unintentional and intentional injuries; suicide; and smoking, alcohol, and marijuana use. Findings of this study indicted that adolescents in both groups report similar rates of risk behaviors in most areas. The differences between the two groups that did exist were few. Driving while drinking occurred more often among adolescents in the D.R. than among adolescents in the U.S. The availability of alcohol 
and lack of drinking-age laws in the D.R. may contribute to this difference. Carrying a weapon was reported to occur more often among adolescents in the U.S. than among adolescents in the D.R. Gang culture in the U.S., particularly in urban areas where minority youth see gang membership as self-preservation may allows not only greater access to weapons but also support the practice of carrying a weapon. More Dominican adolescents in the U.S. reported they tried smoking than their D.R. counterparts. This difference is likely related to the availability and relative cost of cigarettes and may also be a result of the practice of tobacco companies in the U.S. to use aggressive advertising that targets youth. Many of the advertising campaigns promote the notion that it is "cool" to smoke cigarettes. More adolescents in the D.R. reported that they have five or more drinks in a row within the past 30 days compared to adolescents in the U.S. This difference may be due to the education that adolescents in the U.S. receive about the dangers of binge drinking. Alcohol consumption, especially rum manufactured in the D.R., is widespread and begins at a very young age. It is not surprising that there was more reported marijuana use in the U.S. In the experience of the investigators, marijuana and other drugs are rarely available in small towns and campos in the D.R. Since most immigrants to the U.S. initially live in cultural enclaves, it is not surprising that adolescents continue to engage in the kinds of risk behaviors they are familiar with and bring with them.

\section{Limitations}

This study does have some notable limitations. First, data collection sites varied in the D.R. and U.S. In the D.R., data were collected in schools and clinic settings in a town and surrounding rural areas. In the U.S., data collection sites were in community settings such as a local YMCA and street fairs in a large urban area. However, the majority of Dominican immigrants who come to the Boston area actually emigrate from small towns and rural areas in the D.R. Second, this study used 
a non-probability sample, and the D.R. and U.S. subsamples were found to differ in age, grade in school, and gender. Third, data were only collected through self-report measures and only from adolescents who were literate (i.e., able to read either English or Spanish). Limitations not withstanding, this study represented an important step toward obtaining comparative data on a relatively understudied group of adolescents who have behavioral risks that can jeopardize their health and lives.

\section{Conclusion}

A major contribution of this study is that it was the first endeavor to compare risk behaviors of adolescents in the D.R. and their counterparts in the U.S. There is a need for pediatric nurses practitioners, nurses, teachers, counselors and other groups who work with adolescents to understand how culture influences risk taking behaviors. To educate teens regarding the outcomes

of high risk behaviors, it is necessary for providers not only to assume that all adolescents engage in high risk behaviors but also to engage in discussion with adolescents regarding specific risk behaviors that are commonly practiced in their homeland or cultural background. The need for culturally competent care is especially important with immigrant youth who are not only struggling with their identity formation but are doing so in a new culture that attaches different meanings to phenomena and events (Goodenow \& Espin, 1993). Study findings support a great need for ongoing education regarding high risk behaviors with adolescents. 


\section{References}

Banos, R.M., \& Guillen, V. (2000). Psychometric characteristics in normal and social phobic samples for a Spanish version of the Rosenberg self-esteem scale. Psychological Reports, 87, 269-274.

Blake, S. M., Ledsky, R., Goodenow, C., \& O’Donnell, L. (2001). Recency of immigration, substance use, and sexual behavior among Massachusetts adolescents. American Journal of Public Health, 91, 794-798.

Blum, R. W., McNeely, C., \& Nonnemaker, J. (2001). Vulnerability, risk and protection. In B. Fischhoff, E. Nighteingale, \& J. G. Iannotta (Eds.), Adolescent vulnerability: Concepts and measurements (pp. 50-72). Washington, DC: National Academy Press.

Bradley, G., \& Wildman, K. (2002). Psychosocial predictors of emerging adult's risk and reckless behaviors. Journal of Youth and Adolescence, 31(4), 253-265.

Brener, N. D., Collins, J. L., Kann, L., Warren, C. W., \& Williams, B. I. (1995). Reliabiltiy of the Youth Risk Behavior Survey Questionnaire. American Journal of Epidemiology, 141, 575580.

Brener, N. D., Kann, L., Kinchen, S. A., Grunbaum, J. A., Whalen, L., Eaton, D., et al. (2004). Methodology of the Youth Risk Behavior Surveillance System. Morbidity and Mortality Weekly Report, 53(RR-12), 1-13.

Brener, N. D., Kann, L., McManus, T., Kinchen, S. A., Sundberg, M. A., \& Ross, J. G. (2002). Reliability of the 1999 Youth Risk Behavior Survey Questionnaire. Journal of Adolescent Health, 31, 336-342.

Bronfenbrenner, U. (1979). The ecology of human development. Cambridge, MA: Harvard University Press. 
Centers for Disease Control and Prevention. (2004a). DATA2010: The Healthy People 2010 Database--Leading health indicators. Retrieved December 28, 2006, from http://wonder.cdc.gov/data2010/ODLH.HTM

Centers for Disease Control and Prevention. (2004b). Health disparities experienced by Hispanics United States. Morbidity and Mortality Weekly Report, 53, 935-937.

Choi, H. (2002). Understanding adolescent depression in ethno-cultural context. Advances in Nursing Science, 25(2), 71-85.

Currie, C. E. (1998). Health behavior in school-age children: Research protocol for the 1997-1998 survey. Edinburgh, Scotland: World Health Organization Coordinating Center for the Study of Health Behavior in School-Aged Children.

Eaton, D. K., Kann, L., Kinchen, S., Ross, J., Hawkins, J., Harris, W. A., et al. (2006). Youth Risk Behavior Surveillance-United States, 2005. Morbidity and Mortality Weekly Report, 55(SS5), 1-108.

Federation for American Immigration Reform. (2004). City factsheet: Boston, Massachusetts. Retrieved December 28, 2006, from http://www.fairus.org/site/PageServer? pagename=research_researchcc3c_sup

Fleming, J, S., \& Courtney, W.A. (1980). The dimensionality of self-esteem: Some results for a college sample. Journal of Personality and Social Psychology, 5, 921-929

Gabhainn, S. N., \& Francois, Y. (2000). Substance use. In C. Currie, K. Hurrelmann, W. Settertobulte, R. Smith, \& J. Todd (Eds). Health and health behavior among young people (pp. 97-114). Copenhagen, Denmark: World Health Organization Regional Office for Europe. 
Goodenow, C., \& Espin, O. M. (1993). Identity choices in immigrant adolescent females. Adolescence, 28, 173-184.

Hibell, B., Andersson, B., Bjarnasson, T., Ahlstom, S., Balakireva, O., Kokkevi, A., et al. (Eds.) (2003). The ESPAD Report: Alcohol and other drugs among students in 30 European countries. Stockholm, Sweden: Swedish Council for Information on Alcohol and Other Drugs, Pompidou Group at the Council of Europe.

Ingra, V., \& Irwin, C.E. (1996). Theories of adolescent risk-taking behavior. In R. J. DiClemente, W. B. Hansen, \& L. E. Ponton (Eds), Handbook of adolescent risk behavior (pp. 35-53). New York: Plenum.

Jessor, R. (1992). Risk behavior in adolescence: A psychosocial framework for understanding and action. In D. E. Rogers \& E. Ginsberg (Eds), Adolescents at risk: Medical and social perspectives (pp. 19-34). Boulder, CO: Westview.

Joiner, G.W., \& Kashubeck, S. (1996). Acculturation, body image, self-esteem and eating disorder symptomatology in adolescent Mexican American women. Psychology of Women Quarterly, $20,419-425$.

Kann, L., Kinchen, S. A., Williams, B. I., Ross, J. G., Lowry, R., Grunbaum, J. A., et al. (2000). Youth Risk Behavior Surveillance--United States, 1999. Journal of School Health, 70, 271-285.

Le Breton, D. (2004). Risk taking behaviors among young people. Bulletin of the Academy of National Medicine, 188, 1313-1321.

Lightfoot, C. (1997). The culture of adolescent risk-taking. New York: Guilford.

Orshan, S.A. (1999).Acculturation, perceived social support, self-esteem, and pregnancy status among Dominican adolescents. Health Care for Women International, 20, 245-257. 
Ortega, A. N., Rosenheck, R., Alegria, M., \& Desai, R. A. (2000). Acculturation and the lifetime risk of psychiatric and substance use disorders among Hispanics. The Journal of Nervous and Mental Disorders, 188, 728-735.

Perez, M. A., \& Pinzon-Perez, H. (2000). Alcohol, tobacco, and other psychoactive drug use among high school students in Bogota, Columbia. Journal of School Health, 70, 377-380.

Rice, F. P., Dolgin, K. G., \& Dolgin, K. G. (2001). The adolescent: Development, relationships, and culture (10th ed.). Boston: Allyn and Bacon.

Rosenberg, M. (1965). Society and the adolescent self-image. Princeton, NJ: Princeton University Press.

Rosenberg, M. (1989). Society and the Adolescent Self-image. Middletown, CT: Wesleyan University Press.

Statistical Package for the Social Sciences (SPSS, Inc.) (2005). SPSS 14.0 [Computer software]. Chicago, IL: SPSS, Inc.

U.S. Census Bureau. (2004). Releases: Facts for features and special editions. Retrieved January 17, 2007, from http://www.census.gov/Press-Release/ www/releases/archives/facts_for_ features_special_editions/002270.html

Vazsonyi, A.T., Trejos-Castillo, E., \& Huang, L. (2006). Risky sexual behaviors, alcohol use, and drug use: A comparison of eastern and western European adolescents. Journal of Adolscent, 39, 753.e1-753.e11.

Weden, M. M., \& Zabin, L. S. (2005). Gender and ethnic differences in the co-occurence of adolescent risk behaviors. Ethnicity and Health, 10(3), 213-234.

Weinstein, N. D. (1984). Why it won't happen to me: Perceptions of risk factors and 
susceptibility. Health Psychology, 3, 431-457.

Westhoff, W. W., McDermott, R. J., \& Holcomb, D. R. (1996). HIV risk behaviors: A comparison of US Hispanic and Dominican Republic youth. AIDS Education and Prevention, 2, 106-114.

Zuckerman, M. (1979). Beyond the optimal level of arousal. Hillsdale, NJ: Erlbraum. 